\title{
An exponential-trigonometric spline minimizing a seminorm in a Hilbert space
}

\author{
Kholmat M. Shadimetov ${ }^{1,2^{*}}$ and Aziz K. Boltaev
}

\author{
"Correspondence: \\ kholmatshadimetov@mail.ru \\ ${ }^{1}$ V.I. Romanovskiy Institute of \\ Mathematics, Uzbekistan Academy \\ of Sciences, M. Ulugbek street 81, \\ 100170 Tashkent, Uzbekistan \\ ${ }^{2}$ Tashkent Railway Engineering \\ Institute, Odilxojaev street 1, 100167 \\ Tashkent, Uzbekistan
}

\begin{abstract}
In the present paper, using the discrete analogue of the operator $\mathrm{d}^{6} / \mathrm{d} x^{6}-1$, we construct an interpolation spline that minimizes the quantity $\int_{0}^{1}\left(\varphi^{\prime \prime \prime}(x)+\varphi(x)\right)^{2} \mathrm{~d} x$ in the Hilbert space $W_{2}^{(3,0)}$. We obtain explicit formulas for the coefficients of the interpolation spline. The obtained interpolation spline is exact for the exponential-trigonometric functions $e^{-x}, e^{\frac{x}{2}} \cos \left(\frac{\sqrt{3}}{2} x\right)$, and $e^{\frac{x}{2}} \sin \left(\frac{\sqrt{3}}{2} x\right)$.
\end{abstract}

Keywords: Interpolation spline; Hilbert space; The norm minimizing property; Sobolev's method; Discrete argument function

\section{Introduction. Statement of the problem}

One of the problems of approximation is the interpolation problem. The classical method of its solution consists of construction of an interpolation polynomial. However, it is known that the polynomial approximation is unpractical for approximating functions with finite and small smoothness, which often occurs in applications. Therefore, in practice, to approximate functions, splines are used. There are algebraic and variational approaches in the theory of splines [1]. In the algebraic approach, splines are considered as some smooth piecewise polynomial functions. In the variational approach, splines are understood as elements of a Hilbert or Banach space minimizing certain functionals. Then problems of existence, uniqueness, and convergence of splines and algorithms for their constructions are studied based on intrinsic properties of splines (see, e.g., [22]).

$L$-(generalized) splines are a generalization of polynomial splines. Since the results of this paper are connected with $L$-splines, we give some definitions following Ahlberg, Nilson, and Walsh [1].

Let $L$ be a linear differential operator given by

$$
L \equiv a_{m}(x) D^{m}+a_{m-1}(x) D^{m-1}+\cdots+a_{0}(x)
$$

where $D=\mathrm{d} / \mathrm{d} x$, each $a_{j}(x)(j=0,1, \ldots, m)$ is in $C^{m}[a, b]$, and $a_{m}(x) \neq 0$ on $[a, b]$. By $L^{*}$ we denote the formal adjoint of $L$ :

$$
L^{*} \equiv(-1)^{m} D^{m}\left\{a_{m}(x)\right\}+(-1)^{m-1} D^{m-1}\left\{a_{m-1}(x)\right\}+\cdots-D\left\{a_{1}(x)\right\}+a_{0}(x)
$$

(c) The Author(s) 2020. This article is licensed under a Creative Commons Attribution 4.0 International License, which permits use sharing, adaptation, distribution and reproduction in any medium or format, as long as you give appropriate credit to the original author(s) and the source, provide a link to the Creative Commons licence, and indicate if changes were made. The images or other third party material in this article are included in the article's Creative Commons licence, unless indicated otherwise in a credit line to the material. If material is not included in the article's Creative Commons licence and your intended use is not permitted by statutory regulation or exceeds the permitted use, you will need to obtain permission directly from the copyright holder. To view a copy of this licence, visit http://creativecommons.org/licenses/by/4.0/. 
By $K^{m}(a, b)$ we denote the class of all functions $f(x)$ defined on $[a, b]$ that possess absolutely continuous $(n-1)$ th derivatives on $[a, b]$ and have the $n$th derivatives in $L_{2}(a, b)$; it is a Hilbert space with the inner product

$$
\langle f, g\rangle=\int_{a}^{b}(L f)(x) \cdot(L g)(x) \mathrm{d} x
$$

if functions that differ by a solution of the equation $L f=0$ are identified.

If $\Delta: a=x_{0}<x_{1}<\cdots<x_{N}=b$ is a mesh on $[a, b]$, then a generalized spline of deficiency $k(0 \leq k \leq m)$ with respect to $\Delta$ is a function $S_{\Delta}(x)$ from $K^{2 m-k}(a, b)$ that satisfies the differential equation

$$
L^{*} L S_{\Delta}=0
$$

on each open mesh interval $\left(x_{i-1}, x_{i}\right)(i=1,2, \ldots, N)$ of $\Delta$. The ordinary spline of deficiency 1 allows discontinuities in the $(2 m-1)$ th derivative, but only at mesh points.

From the results of Ahlberg, Nilson, and Walsh [1] ti is known that for generalized spline of deficiency 1, the following is true: Let $\Delta: a=x_{0}<x_{1}<\cdots<x_{N}=b$ and $Y=$ $\left\{y_{i}, i=0,1, \ldots, N\right\}$ be given. Then among all functions $f(x)$ in $K^{m}(a, b)$ such that $f\left(x_{i}\right)=y_{i}$ $(i=0,1, \ldots, N)$, the generalized spline $S_{\Delta}(Y ; x)$, when it exists, minimizes the quantity $\int_{a}^{b}(L f(x))^{2} \mathrm{~d} x$.

Further, we give some results obtained from the theory of $L$-splines. First, contributions to the theory of splines include the works of Greville, Ahlberg, Nilson, and Walsh and of Schultz and Varga (see [22, p. 459]). These works are concentrated on natural $L$-splines, which appear as solutions to the corresponding best interpolation problems, and the order of approximation of generalized splines was first studied.

Trigonometric splines were first considered by Schoenberg [20]. Construction of local $B$-splines for general spaces of $L$-splines was studied in $[9,14]$. Further results on the of approximation of trigonometric splines were obtained in [16] based on construction of quasi-interpolation operators. In [12], control curves of trigonometric splines are constructed, and it is proved that they have similar properties as polynomial splines. Then trigonometric $B$-splines were studied in $[8,10,19]$.

The space of natural hyperbolic splines was introduced in [22, p. 407]. In [21], it is shown that hyperbolic splines can be treated as an example of $L$-splines.

There are different variants of hyperbolic and trigonometric $L$-splines. Recently, several classwa of splines whose parts are combinations of algebraic polynomials and exponential or trigonometric functions are considered. Splines whose parts are spanned by $\{1, x, \exp (\rho x), \exp (-\rho x)\}$ are called tension exponential splines. Such splines were studied in [11, 17-19]. Algebraic and exponential $B$-splines generated over the space spanned by $\left\{1, x, \ldots, x^{m-3}, \cosh x, \sinh x\right\}$ were presented in [15]. Algebraic-trigonometric splines generated by $\{1, x, \sin x, \cos x\}$ were studied in $[31,32]$. Similar results were developed in $[13,30]$ for splines obtained by combinations of functions $\left\{1, x, \ldots, x^{m-3}, \cos x, \sin x\right\}$. In [5], using Sobolev's method, interpolation splines that minimize the expression $\int_{0}^{1}\left(\varphi^{(m)}+\right.$ $\left.\omega^{2} \varphi^{(m-2)}(x)\right)^{2} \mathrm{~d} x$ in the space $K_{2}\left(P_{m}\right)$ are constructed. Explicit formulas for the coefficients of the interpolation splines are obtained. The obtained interpolation splines are exact for monomials $1, x, x^{2}, \ldots, x^{m-3}$ and for trigonometric functions $\sin \omega x$ and $\cos \omega x$. 
In the present work, we solve the problem of construction of a natural $L$-spline in the case $m=3, L \equiv \mathrm{d}^{3} / \mathrm{d} x^{3}+1$ on the interval $[0,1]$, and we denote by $W_{2}^{(3,0)}(0,1)$ the corresponding space, that is,

$$
W_{2}^{(3,0)}(0,1)=\left\{\varphi:[0,1] \rightarrow R \mid \varphi^{\prime \prime} \text { is absolutely continuous, and } \varphi^{\prime \prime \prime} \in L_{2}(0,1)\right\}
$$

The class $W_{2}^{(3,0)}$, equipped with the seminorm

$$
\|\varphi\|_{W_{2}^{(3,0)}}=\left(\int_{0}^{1}\left(\varphi^{\prime \prime \prime}(x)+\varphi(x)\right)^{2} \mathrm{~d} x\right)^{1 / 2}
$$

is a Hilbert space if we identify functions that differ by a solution of the equation $\varphi^{\prime \prime \prime}(x)+$ $\varphi(x)=0$. A solution of the last equation has the form $\varphi(x)=d_{0} e^{-x}+d_{1} e^{\frac{x}{2}} \cdot \cos \left(\frac{\sqrt{3}}{2} x\right)+d_{2} e^{\frac{x}{2}}$. $\sin \left(\frac{\sqrt{3}}{2} x\right)$.

Suppose we are given the values $y_{\beta}, \beta=0,1, \ldots, N$, at points $x_{\beta} \in[0,1], \beta=0,1, \ldots, N$. Consider the following variational interpolation problem.

Problem 1 Among all functions $f(x)$ in $W_{2}^{(3,0)}$ satisfying the conditions

$$
f\left(x_{\beta}\right)=\varphi\left(x_{\beta}\right), \quad \beta=0,1, \ldots, N,
$$

find a function $S(x)$ that gives the minimum of the norm (2), where $x_{\beta} \in[0,1]$ are the nodes of interpolation, and $\varphi\left(x_{\beta}\right)=y_{\beta}$ are given values.

The solution $S(x)$ of Problem 1 is a ordinary generalized spline and is uniquely defined with respect to mesh $\Delta: 0=x_{0}<x_{1}<\cdots<x_{N}=1$ on the interval $[0,1]$ as follows:

(i) $S(x)$ is a linear combination of functions $e^{-x}, e^{\frac{x}{2}} \cos \left(\frac{\sqrt{3}}{2} x\right), e^{\frac{x}{2}} \sin \left(\frac{\sqrt{3}}{2} x\right), e^{x}$, $e^{-\frac{x}{2}} \cos \left(\frac{\sqrt{3}}{2} x\right)$, and $e^{-\frac{x}{2}} \sin \left(\frac{\sqrt{3}}{2} x\right)$ on each open mesh interval $\left(x_{\beta}, x_{\beta+1}\right)$, $\beta=0,1, \ldots, N-1$;

(ii) $S(x)$ is a linear combination of functions $e^{-x}, e^{\frac{x}{2}} \cos \left(\frac{\sqrt{3}}{2} x\right)$, and $e^{\frac{x}{2}} \sin \left(\frac{\sqrt{3}}{2} x\right)$ on the intervals $(-\infty, 0)$ and $(1, \infty)$;

(iii) $S^{(\alpha)}\left(x_{\beta}^{-}\right)=S^{(\alpha)}\left(x_{\beta}^{+}\right), \alpha=0,1, \ldots, 4$ and $\beta=1,2, \ldots, N-1$;

(iv) $S\left(x_{\beta}\right)=\varphi\left(x_{\beta}\right), \beta=0,1, \ldots, N$;

(v) $S(x)$ satisfies the following boundary conditions:

$$
\begin{aligned}
& S^{\prime \prime \prime}(1)+S(1)=0, \quad S^{\prime \prime \prime}(0)+S(0)=0, \\
& S^{(4)}(1)+S^{\prime}(1)=0, \quad S^{(4)}(0)+S^{\prime}(0)=0 .
\end{aligned}
$$

We consider the fundamental solution

$$
G(x)=\frac{\operatorname{sgn} x}{6} \cdot\left[\sinh (x)+e^{\frac{x}{2}} \cos \left(\frac{\sqrt{3}}{2} x+\frac{\pi}{3}\right)+e^{-\frac{x}{2}} \cos \left(\frac{\sqrt{3}}{2} x+\frac{2 \pi}{3}\right)\right]
$$

of the differential operator $\frac{\mathrm{d}^{6}}{\mathrm{~d} x^{6}}-1$, that is, the solution of the equation

$$
G^{(6)}(x)-G(x)=\delta(x)
$$

where $\delta(x)$ is the Dirac delta-function. 
Remark The following rule for finding a fundamental solution of a linear differential operator

$$
P\left(\frac{\mathrm{d}}{\mathrm{d} x}\right):=\frac{\mathrm{d}^{m}}{\mathrm{~d} x^{m}}+a_{1} \frac{\mathrm{d}^{m-1}}{\mathrm{~d} x^{m-1}}+\cdots+a_{m}
$$

where $a_{j}$ are real numbers, is given in [29, pp. 88-89]: replacing $\frac{\mathrm{d}}{\mathrm{d} x}$ by $p$, we get a polynomial $P(p)$. Then we expand the expression $\frac{1}{P(p)}$ to partial fractions:

$$
\frac{1}{P(p)}=\prod_{j}(p-\lambda)^{-k_{j}}=\sum_{j}\left[c_{j, k_{j}}(p-\lambda)^{-k_{j}}+\cdots+c_{j, 1}(p-\lambda)^{-1}\right]
$$

and with every partial fraction $(p-\lambda)^{-k}$, we associate $\frac{x^{k-1} \operatorname{sgn} x}{2(k-1) !} \cdot e^{\lambda x}$.

Using this rule, we find the function $G(x)$ that is the fundamental solution of the operator $\frac{\mathrm{d}^{6}}{\mathrm{~d} x^{6}}-1$ and has the form (4).

It is easy to check that the fifth derivative of the function

$$
\begin{aligned}
G\left(x-x_{\gamma}\right)= & \frac{\operatorname{sgn}\left(x-x_{\gamma}\right)}{6} \cdot\left[\sinh \left(x-x_{\gamma}\right)+e^{\frac{x-x_{\gamma}}{2}} \cdot \cos \left(\frac{\sqrt{3}}{2}\left(x-x_{\gamma}\right)+\frac{\pi}{3}\right)\right. \\
& \left.+e^{-\frac{x-x_{\gamma}}{2}} \cdot \cos \left(\frac{\sqrt{3}}{2}\left(x-x_{\gamma}\right)+\frac{2 \pi}{3}\right)\right]
\end{aligned}
$$

has a discontinuity equal to 1 at the point $x_{\gamma}$, and the third and the fours derivatives of $G\left(x-x_{\gamma}\right)$ are continuous. Suppose a function $p_{\gamma}(x)$ coincides with the spline $S(x)$ on the interval $\left(x_{\gamma}, x_{\gamma+1}\right)$, that is, $p_{\gamma}(x):=p_{\gamma-1}(x)+C_{\gamma} G\left(x-x_{\gamma}\right), x \in\left(x_{\gamma}, x_{\gamma+1}\right)$, where $C_{\gamma}$ is the jump of the function $S^{(5)}(x)$ at $x_{\gamma}$ :

$$
C_{\gamma}=S^{(5)}\left(x_{\gamma}^{+}\right)-S^{(5)}\left(x_{\gamma}^{-}\right)
$$

Then the spline $S(x)$ can be written in the form

$$
S(x)=\sum_{\gamma=0}^{N} C_{\gamma} G\left(x-x_{\gamma}\right)+p_{-1}(x),
$$

where $p_{-1}(x)=d_{0} e^{-x}+d_{1} e^{\frac{x}{2}} \cdot \cos \left(\frac{\sqrt{3}}{2} x\right)+d_{2} e^{\frac{x}{2}} \cdot \sin \left(\frac{\sqrt{3}}{2} x\right)$, and $d_{0}, d_{1}$, and $d_{2}$ are real numbers. It is known that (see, e.g., [28]) the solution $S(x)$ of the form (4) of Problem 1 exists and is unique when $N \geq 2$, and the coefficients $C_{\gamma}, d_{0}, d_{1}$, and $d_{2}$ of $S(x)$ are determined by the following system of $N+4$ linear equations:

$$
\begin{aligned}
& \sum_{\gamma=0}^{N} C_{\gamma} G\left(x_{\beta}-x_{\gamma}\right)+d_{0} e^{-x_{\beta}}+d_{1} e^{\frac{x_{\beta}}{2}} \cos \left(\frac{\sqrt{3}}{2} x_{\beta}\right) \\
& +d_{2} e^{\frac{x_{\beta}}{2}} \sin \left(\frac{\sqrt{3}}{2} x_{\beta}\right)=\varphi\left(x_{\beta}\right), \quad \beta=0,1, \ldots, N, \\
& \sum_{\gamma=0}^{N} C_{\gamma} e^{-x_{\gamma}}=0,
\end{aligned}
$$




$$
\begin{aligned}
& \sum_{\gamma=0}^{N} C_{\gamma} e^{\frac{x_{\gamma}}{2}} \cos \left(\frac{\sqrt{3}}{2} x_{\gamma}\right)=0, \\
& \sum_{\gamma=0}^{N} C_{\gamma} e^{\frac{x_{\gamma}}{2}} \sin \left(\frac{\sqrt{3}}{2} x_{\gamma}\right)=0 .
\end{aligned}
$$

It is easy to show that the spline $S(x)$ defined by equation (8) with coefficients $C_{\gamma}, d_{0}, d_{1}$, and $d_{2}$ satisfies conditions (i) $-(\mathrm{v})$.

The main aim of the present paper is to solve Problem 1 , that is, to solve system (9)(12) for equally spaced nodes $x_{\beta}=h \beta, \beta=0,1, \ldots, N, h=1 / N, N \geq 2$, and to find analytic formulas for the coefficients $C_{\gamma}, d_{0}, d_{1}$, and $d_{2}$ of $S(x)$.

It should be noted that using Sobolev method, interpolation splines minimizing the seminorms in different Hilbert spaces were constructed in [2, 4, 6, 7, 23, 24]. Furthermore, connection between interpolation splines and optimal quadrature formulas in the sense of Sard in $L_{2}^{(m)}(0,1)$ and $K_{2}\left(P_{2}\right)$ spaces were shown in [4] and [6].

The rest of the paper is organized as follows. In Sect. 2, we give some definitions and known results. In Sect. 3, we give an algorithm for solving system (9)-(12) when the nodes $x_{\beta}$ are equally spaced. Using this algorithm, the coefficients of the interpolation spline $S(x)$ are computed in Sect. 4.

\section{Preliminaries}

In this section, we give some definitions and known results that we need to prove the main results.

We mainly use the concept of discrete argument functions and operations on them. The theory of discrete argument functions is given in $[25,27]$. For completeness, we give some definitions about functions of discrete argument.

Assume that the nodes $x_{\beta}$ are equally spaced, that is, $x_{\beta}=h \beta, h=\frac{1}{N}, N=1,2, \ldots$. Further, we also use the notation $[\beta]=h \beta$.

Definition 1 The function $\varphi[\beta]$ is a function of discrete argument if it is given on some set of integer values of $\beta$.

Definition 2 The inner product of two discrete-argument functions $\varphi[\beta]$ and $\psi[\beta]$ is given by

$$
[\varphi[\beta], \psi[\beta]]=\sum_{\beta=-\infty}^{\infty} \varphi[\beta] \cdot \psi[\beta],
$$

if the series on the right-hand side of the last equality converges absolutely.

Definition 3 The convolution of two functions $\varphi[\beta]$ and $\psi[\beta]$ is the inner product

$$
\varphi[\beta] * \psi[\beta]=[\varphi[\gamma], \psi[\beta-\gamma]]=\sum_{\gamma=-\infty}^{\infty} \varphi[\gamma] \cdot \psi[\beta-\gamma] .
$$

In our computations we need the discrete analogue $D[\beta]$ of the differential operator $\frac{\mathrm{d}^{6}}{\mathrm{~d} x^{6}}-1$, which satisfies the equality

$$
D[\beta] * G[\beta]=\delta[\beta],
$$


where $G[\beta]$ is the discrete-argument function corresponding to $G(x)$ defined by $(4), \delta[\beta]$ is equal to 0 when $\beta \neq 0$ and to 1 when $\beta=0$, that is, $\delta[\beta]$ is the discrete delta-function. Equation (13) is a discrete analogue of equation (5).

In $[2,3]$ the discrete analogue $D[\beta]$ of the differential operator $\frac{\mathrm{d}^{6}}{\mathrm{~d} x^{6}}-1$, which satisfies equation (13), was constructed, and the following theorem was proved.

Theorem 1 The discrete analogue of the differential operator $\frac{\mathrm{d}^{6}}{\mathrm{~d} x^{6}}-1$ satisfying equation (13) has the form

$$
D[\beta]=\frac{3}{K} \begin{cases}\sum_{k=1}^{2} B_{k} \lambda_{k}^{|\beta|-1}, & |\beta| \geq 2, \\ 1+\sum_{k=1}^{2} B_{k}, & |\beta|=1, \\ K_{3}-K_{1}+\sum_{k=1}^{2} \frac{B_{k}}{\lambda_{k}}, & \beta=0,\end{cases}
$$

where

$$
\begin{aligned}
& K= \sinh (h)+\sinh \left(\frac{h}{2}\right) \cdot \cos \left(\frac{\sqrt{3}}{2} h\right)-\sqrt{3} \cdot \cosh \left(\frac{h}{2}\right) \cdot \sin \left(\frac{\sqrt{3}}{2} h\right), \\
& K_{1}= 2 \cosh (h) \\
&+\frac{4 \cos \left(\frac{\sqrt{3}}{2} h\right) \cosh \left(\frac{h}{2}\right) \sinh (h)+\sinh (h)-\sqrt{3} \sin (\sqrt{3} h)-2 \sinh (h) \cosh (h)}{\sinh (h)+\sinh \left(\frac{h}{2}\right) \cdot \cos \left(\frac{\sqrt{3}}{2} h\right)-\sqrt{3} \cdot \cosh \left(\frac{h}{2}\right) \cdot \sin \left(\frac{\sqrt{3}}{2} h\right)} \\
& K_{2}=2+\frac{2 \cos (\sqrt{3} h) \sinh (h)+4 \sinh (h) \cosh (h)-2 \sqrt{3} \sin (\sqrt{3} h) \cosh (h)}{\sinh (h)+\sinh \left(\frac{h}{2}\right) \cdot \cos \left(\frac{\sqrt{3}}{2} h\right)-\sqrt{3} \cdot \cosh \left(\frac{h}{2}\right) \cdot \sin \left(\frac{\sqrt{3}}{2} h\right)}, \\
& K_{3}=2 \cdot\left(\cosh (h)+2 \cos \left(\frac{\sqrt{3}}{2} h\right) \cosh \left(\frac{h}{2}\right)\right) \\
& B_{k}=\frac{\left(\lambda_{k}^{2}-2 \lambda_{k} \cdot \cosh (h)+1\right) \cdot A_{4}\left(\lambda_{k}\right)}{\left(\lambda_{k}^{2}-1\right) \cdot\left(2 \lambda_{k}^{2}-K_{1} \lambda_{k}+2\right)}, \\
& A_{4}\left(\lambda_{k}\right)=\lambda_{k}^{4}-4 \lambda_{k}^{3} \cdot \cos \left(\frac{\sqrt{3}}{2} h\right) \cosh \left(\frac{h}{2}\right)+2 \lambda_{k}^{2} \cdot(1+\cos (\sqrt{3} h)+\cosh (h)) \\
& \quad-4 \lambda_{k} \cdot \cos \left(\frac{\sqrt{3}}{2} h\right) \cosh \left(\frac{h}{2}\right)+1, \\
& \lambda_{k}=\frac{1}{4} \cdot\left[K_{1}+\sqrt{K_{1}^{2}-4 \cdot K_{2}+8}+(-1)^{k} \sqrt{\left.\left(K_{1}+\sqrt{K_{1}^{2}-4 \cdot K_{2}+8}\right)^{2}-16\right], \quad k=1,2,}\right.
\end{aligned}
$$

$h$ is a small positive parameter, and $\lambda_{k}$ are the roots of the polynomial $A_{4}(\lambda)$ such that $\left|\lambda_{k}\right|<1$.

Furthermore, several properties of the discrete-argument function $D[\beta]$ were given in $[2,3]$. Here we give the following its properties, which we need in our computations.

Theorem 2 The discrete analogue $D[\beta]$ of the differential operator $\frac{\mathrm{d}^{6}}{\mathrm{~d} x^{6}}-1$ satisfies the following equalities:

(1) $D[\beta] * e^{[\beta]}=0$,

(2) $D[\beta] * e^{-[\beta]}=0$,

(3) $D[\beta] * e^{\frac{[\beta]}{2}} \cdot \cos \left(\frac{\sqrt{3}}{2}[\beta]\right)=0$, 
(4) $D[\beta] * e^{\frac{[\beta]}{2}} \cdot \sin \left(\frac{\sqrt{3}}{2}[\beta]\right)=0$,

(5) $D[\beta] * e^{-\frac{[\beta]}{2}} \cdot \cos \left(\frac{\sqrt{3}}{2}[\beta]\right)=0$,

(6) $D[\beta] * e^{-\frac{[\beta]}{2}} \cdot \sin \left(\frac{\sqrt{3}}{2}[\beta]\right)=0$.

\section{The algorithm for computation of coefficients of interpolation spline}

In the present section, we give an algorithm for solving system (9)-(12) when the nodes $x_{\beta}$ are equally spaced, that is, $x_{\beta}=h \beta, h=\frac{1}{N}, N=1,2, \ldots$. Here we use a similar method suggested by Sobolev $[26,27]$ for finding the coefficients of optimal quadrature formulas in the Sobolev space $L_{2}^{(m)}(0,1)$. We note that here $[\beta]$ means $(h \beta)$.

Suppose that $C[\beta]=0$ when $\beta<0$ and $\beta>N$. Using Definition 3, we rewrite system (9)-(12) in the convolution form:

$$
\begin{aligned}
& G[\beta] * C[\beta]+d_{0} e^{-[\beta]}+d_{1} e^{\frac{[\beta]}{2}} \cdot \cos \left(\frac{\sqrt{3}}{2}[\beta]\right) \\
& +d_{2} e^{\frac{[\beta]}{2}} \cdot \sin \left(\frac{\sqrt{3}}{2}[\beta]\right)=\varphi[\beta], \quad \beta=0,1, \ldots, N, \\
& \sum_{\beta=0}^{N} C[\beta] \cdot e^{-[\beta]}=0, \\
& \sum_{\beta=0}^{N} C[\beta] \cdot e^{\frac{[\beta]}{2}} \cdot \cos \left(\frac{\sqrt{3}}{2}[\beta]\right)=0, \\
& \sum_{\beta=0}^{N} C[\beta] \cdot e^{\frac{[\beta]}{2}} \cdot \sin \left(\frac{\sqrt{3}}{2}[\beta]\right)=0 .
\end{aligned}
$$

Thus we have the following problem.

Problem 2 Find the coefficients $C[\beta](\beta=0,1, \ldots, N), d_{0}, d_{1}$, and $d_{2}$ that satisfy system (15)-(18).

Further, we investigate Problem 2, which is equivalent to Problem 1. Instead of $C[\beta]$, we introduce the following discrete-argument functions:

$$
\begin{aligned}
& v[\beta]=G[\beta] * C[\beta], \\
& u[\beta]=v[\beta]+d_{0} e^{-[\beta]}+d_{1} e^{\frac{[\beta]}{2}} \cdot \cos \left(\frac{\sqrt{3}}{2}[\beta]\right)+d_{2} e^{\frac{[\beta]}{2}} \cdot \sin \left(\frac{\sqrt{3}}{2}[\beta]\right) .
\end{aligned}
$$

Now we express the coefficients $C[\beta]$ by the function $u[\beta]$.

Taking into account (14), (20), and Theorems 1 and 2, for the coefficients, we have

$$
C[\beta]=D[\beta] * u[\beta]
$$

Thus, if we find the function $u[\beta]$, then the coefficients $C[\beta]$ will be found from equality (21)

To calculate convolution (21), it is required to find the representation of the function $u[\beta]$ for all integer values of $\beta$. From equality (15) we get that $u[\beta]=\varphi[\beta]$ when $[\beta] \in[0,1]$. Now we need to find the representation of the function $u[\beta]$ when $\beta<0$ and $\beta>N$. 
Since $C[\beta]=0$ when $[\beta] \notin[0,1]$, we have

$$
C[\beta]=D[\beta] * u[\beta]=0 \quad \text { for }[\beta] \notin[0,1]
$$

Now we calculate the convolution $v[\beta]=G[\beta] * C[\beta]$ when $\beta \leq 0$ and $\beta \geq N$.

Suppose $\beta \leq 0$. Then taking into account equalities (8) and (16)-(18), we have

$$
\begin{aligned}
v[\beta]= & \sum_{\gamma=-\infty}^{\infty} C[\gamma] G[\beta-\gamma] \\
= & -\frac{1}{12}\left[-e^{-[\beta]} \sum_{\gamma=0}^{N} C[\gamma] e^{[\gamma]}\right. \\
& +\left(\sum_{\gamma=0}^{N} C[\gamma] e^{-\frac{[\gamma]}{2}} \cos \left(\frac{\sqrt{3}}{2}[\gamma]\right)+\sqrt{3} \sum_{\gamma=0}^{N} C[\gamma] e^{-\frac{[\gamma]}{2}} \sin \left(\frac{\sqrt{3}}{2}[\gamma]\right)\right) \\
& \times e^{\frac{[\beta]}{2}} \cos \left(\frac{\sqrt{3}}{2}[\beta]\right) \\
& +\left(\sum_{\gamma=0}^{N} C[\gamma] e^{-\frac{[\gamma]}{2}} \sin \left(\frac{\sqrt{3}}{2}[\gamma]\right)-\sqrt{3} \sum_{\gamma=0}^{N} C[\gamma] e^{-\frac{[\gamma]}{2}} \cos \left(\frac{\sqrt{3}}{2}[\gamma]\right)\right) \\
& \left.\times e^{\frac{[\beta]}{2}} \sin \left(\frac{\sqrt{3}}{2}[\beta]\right)\right] .
\end{aligned}
$$

Thus, when $\beta \leq 0$, we get

$$
v[\beta]=-b_{0} e^{-[\beta]}-b_{1} e^{\frac{[\beta]}{2}} \cos \left(\frac{\sqrt{3}}{2}[\beta]\right)-b_{2} e^{\frac{[\beta]}{2}} \sin \left(\frac{\sqrt{3}}{2}[\beta]\right),
$$

where

$$
\begin{aligned}
& b_{0}=-\frac{1}{12} \sum_{\gamma=0}^{N} C[\gamma] e^{[\gamma]}, \\
& b_{1}=\frac{1}{12} \sum_{\gamma=0}^{N} C[\gamma] e^{-\frac{[\gamma]}{2}}\left[\cos \left(\frac{\sqrt{3}}{2}[\gamma]\right)+\sqrt{3} \sin \left(\frac{\sqrt{3}}{2}[\gamma]\right)\right],
\end{aligned}
$$

and

$$
b_{2}=\frac{1}{12} \sum_{\gamma=0}^{N} C[\gamma] e^{-\frac{[\gamma]}{2}}\left[\sin \left(\frac{\sqrt{3}}{2}[\gamma]\right)-\sqrt{3} \cos \left(\frac{\sqrt{3}}{2}[\gamma]\right)\right] \text {. }
$$

Similarly, in the case $\beta \geq N$, for the convolution $v[\beta]=G[\beta] * C[\beta]$, we obtain

$$
v[\beta]=b_{0} e^{-[\beta]}+b_{1} e^{\frac{[\beta]}{2}} \cos \left(\frac{\sqrt{3}}{2}[\beta]\right)+b_{2} e^{\frac{[\beta]}{2}} \sin \left(\frac{\sqrt{3}}{2}[\beta]\right) .
$$

We denote

$$
d_{0}^{-}=d_{0}-b_{0}, \quad d_{1}^{-}=d_{1}-b_{1}, \quad d_{2}^{-}=d_{2}-b_{2},
$$




$$
d_{0}^{+}=d_{0}+b_{0}, \quad d_{1}^{+}=d_{1}+b_{1}, \quad d_{2}^{+}=d_{2}+b_{2},
$$

Taking into account (20), (22), and (24), we get the following problem.

Problem 3 Find the solution of the equation

$$
D[\beta] * u[\beta]=0, \quad[\beta] \notin[0,1]
$$

of the form

$$
u[\beta]= \begin{cases}d_{0}^{-} e^{-[\beta]}+d_{1}^{-} e^{\frac{[\beta]}{2}} \cos \left(\frac{\sqrt{3}}{2}[\beta]\right)+d_{2}^{-} e^{\frac{[\beta]}{2}} \sin \left(\frac{\sqrt{3}}{2}[\beta]\right), & \beta \leq 0, \\ \varphi[\beta], & 0 \leq \beta \leq N, \\ d_{0}^{+} e^{-[\beta]}+d_{1}^{+} e^{\frac{[\beta]}{2}} \cos \left(\frac{\sqrt{3}}{2}[\beta]\right)+d_{2}^{+} e^{\frac{[\beta]}{2}} \sin \left(\frac{\sqrt{3}}{2}[\beta]\right), & \beta \geq N .\end{cases}
$$

If we find $d_{0}^{-}, d_{0}^{+}, d_{1}^{-}, d_{1}^{+}$, and $d_{2}^{-}, d_{2}^{+}$, then from (25) and (26) we have

$$
\begin{aligned}
& d_{n}=\frac{1}{2}\left(d_{n}^{+}+d_{n}^{-}\right), \quad n=0,1,2, \\
& b_{n}=\frac{1}{2}\left(b_{n}^{+}-b_{n}^{-}\right), \quad n=0,1,2 .
\end{aligned}
$$

The unknowns $d_{0}^{-}, d_{0}^{+}, d_{1}^{-}, d_{1}^{+}$, and $d_{2}^{-}, d_{2}^{+}$can be found from equation (27) using the function $D[\beta]$ defined by (14). Then we obtain an explicit form of the function $u[\beta]$, and from (21) we find the coefficients $C[\beta]$. Furthermore, from (29) we get $d_{0}, d_{1}$, and $d_{2}$.

Thus Problem 3 and, respectively, Problems 2 and 1 will be solved.

In the next section, we apply this algorithm to compute the coefficients $C[\beta], \beta=$ $0,1, \ldots, N, d_{0}, d_{1}$, and $d_{2}$ of the interpolation spline (8) for $N \geq 2$.

\section{Computation of coefficients of interpolation spline (8)}

In this section, using the presented algorithm, we obtain explicit formulas for the coefficients of the interpolation spline (8), which, as we have proved in the previous section, is the solution of Problem 1.

It should be noted that the interpolation spline (8), the solution of Problem 1, is exact for functions $e^{-x}, e^{\frac{x}{2}} \cos \left(\frac{\sqrt{3}}{2} x\right)$, and $e^{\frac{x}{2}} \sin \left(\frac{\sqrt{3}}{2} x\right)$.

We now obtain exact formulas for the coefficients of the interpolation spline (8) in the following:

Theorem 3 The coefficients of the interpolation spline (8) with equally spaced nodes in the space $W_{2}^{(3,0)}$ have the following form:

$$
\begin{aligned}
C[0]= & \frac{3}{K}\left[\varphi(0)\left(K_{3}-K_{1}\right)+\varphi(h)+d_{0}^{-} e^{h}+d_{1}^{-} e^{-\frac{h}{2}} \cos \left(\frac{\sqrt{3}}{2} h\right)-d_{2}^{-} e^{-\frac{h}{2}} \sin \left(\frac{\sqrt{3}}{2} h\right)\right. \\
& \left.+\sum_{k=1}^{2} \frac{B_{k}}{\lambda_{k}}\left(\sum_{\gamma=0}^{N} \lambda_{k}^{\gamma} \varphi(h \gamma)+M_{k}+\lambda_{k}^{N} \cdot N_{k}\right)\right],
\end{aligned}
$$




$$
\begin{aligned}
& C[\beta]= \frac{3}{K}\left[\varphi(h \beta)\left(K_{3}-K_{1}\right)+\varphi(h(\beta-1))+\varphi(h(\beta+1))\right. \\
&\left.+\sum_{k=1}^{2} \frac{B_{k}}{\lambda_{k}}\left(\sum_{\gamma=0}^{N} \lambda_{k}^{|\beta-\gamma|} \varphi(h \gamma)+\lambda_{k}^{\beta} \cdot M_{k}+\lambda_{k}^{N-\beta} \cdot N_{k}\right)\right], \quad \beta=1,2, \ldots, N-1, \\
& C[N]= \frac{3}{K}\left[\varphi(1)\left(K_{3}-K_{1}\right)+\varphi(1-h)+d_{0}^{+} e^{-(1+h)}+d_{1}^{+} e^{\frac{1+h}{2}} \cos \left(\frac{\sqrt{3}}{2}(1+h)\right)\right. \\
&\left.+d_{2}^{+} e^{\frac{1+h}{2}} \sin \left(\frac{\sqrt{3}}{2}(1+h)\right)+\sum_{k=1}^{2} \frac{B_{k}}{\lambda_{k}}\left(\sum_{\gamma=0}^{N} \lambda_{k}^{N-\gamma} \varphi(h \gamma)+\lambda_{k}^{N} \cdot M_{k}+N_{k}\right)\right], \\
& d_{n}=\frac{1}{2}\left(d_{n}^{+}+d_{n}^{-}\right), \quad n=0,1,2,
\end{aligned}
$$

where

$$
\begin{aligned}
M_{k}= & \lambda_{k}\left[\frac{d_{0}^{-} e^{h}}{1-\lambda_{k} e^{h}}-\frac{d_{1}^{-}\left(\lambda_{k}-e^{\frac{h}{2}} \cos \left(\frac{\sqrt{3}}{2} h\right)\right)+d_{2}^{-} e^{\frac{h}{2}} \sin \left(\frac{\sqrt{3}}{2} h\right)}{\lambda_{k}^{2}-2 \lambda_{k} e^{\frac{h}{2}} \cos \left(\frac{\sqrt{3}}{2} h\right)+e^{h}}\right], \\
N_{k}= & \lambda_{k}\left[\frac{d_{0}^{+} e^{-1}}{e^{h}-\lambda_{k}}+e^{\frac{1+h}{2}}\left[\frac{d_{1}^{+}\left(\cos \left(\frac{\sqrt{3}}{2}(1+h)\right)-\lambda_{k} e^{\frac{h}{2}} \cos \left(\frac{\sqrt{3}}{2}\right)\right)}{\lambda_{k}^{2} e^{h}-2 \lambda_{k} e^{\frac{h}{2}} \cos \left(\frac{\sqrt{3}}{2} h\right)+1}\right.\right. \\
& \left.\left.+\frac{d_{2}^{+}\left(\sin \left(\frac{\sqrt{3}}{2}(1+h)\right)-\lambda_{k} e^{\frac{h}{2}} \sin \left(\frac{\sqrt{3}}{2}\right)\right)}{\lambda_{k}^{2} e^{h}-2 \lambda_{k} e^{\frac{h}{2}} \cos \left(\frac{\sqrt{3}}{2} h\right)+1}\right]\right], \quad k=1,2,
\end{aligned}
$$

and $K, K_{1}, K_{3}, B_{k}$, and $\lambda_{k}$ are defined in Theorem $1,\left|\lambda_{k}\right|<1, d_{n}^{-}$and $d_{n}^{+}, n=0,1,2$, are defined from system (32)-(33), (35)-(38).

Proof First, we find the expressions for $d_{0}^{-}$and $d_{0}^{+}$. When $\beta=0$ and $\beta=N$, from (28) for $d_{0}^{-}$and $d_{0}^{+}$we get

$$
\begin{aligned}
& d_{0}^{-}=\varphi(0)-d_{1}^{-}, \\
& d_{0}^{+}=e \cdot \varphi(1)-e^{\frac{3}{2}} \cos \frac{\sqrt{3}}{2} d_{1}^{+}-e^{\frac{3}{2}} \sin \frac{\sqrt{3}}{2} d_{2}^{+} .
\end{aligned}
$$

Now we have four unknowns $d_{1}^{-}, d_{2}^{-}, d_{1}^{+}$, and $d_{2}^{+}$.

From equation (27) for $\beta=-1,-2$ and $\beta=N+1, N+2$, we find $d_{1}^{-}, d_{2}^{-}, d_{1}^{+}$, and $d_{2}^{+}$.

Taking into account (29), (32), and (33), from (27) we get the following equation for $d_{1}^{-}$, $d_{2}^{-}, d_{1}^{+}$, and $d_{2}^{+}$:

$$
\begin{aligned}
& \sum_{\gamma=-\infty}^{-1} D[\beta-\gamma]\left[d_{0}^{-} e^{-[\beta]}+d_{1}^{-} e^{\frac{[\beta]}{2}} \cos \left(\frac{\sqrt{3}}{2}[\beta]\right)+d_{2}^{-} e^{\frac{[\beta]}{2}} \sin \left(\frac{\sqrt{3}}{2}[\beta]\right)\right] \\
& +\sum_{\gamma=0}^{N} D[\beta-\gamma] \varphi[\gamma]+\sum_{\gamma=N+1}^{\infty} D[\beta-\gamma]\left[d_{0}^{+} e^{-[\beta]}+d_{1}^{+} e^{\frac{[\beta]}{2}} \cos \left(\frac{\sqrt{3}}{2}[\beta]\right)\right. \\
& \left.+d_{2}^{+} e^{\frac{[\beta]}{2}} \sin \left(\frac{\sqrt{3}}{2}[\beta]\right)\right]=0
\end{aligned}
$$


where $\beta<0$ and $\beta>N$. First, we consider the cases $\beta=-1,-2$. From (34) we obtain the following system of two linear equations

$$
\begin{aligned}
& \sum_{\gamma=1}^{\infty} D {[\gamma-1] \cdot\left[d_{1}^{-}\left(e^{-\frac{[\gamma]}{2}} \cos \left(\frac{\sqrt{3}}{2}[\gamma]\right)-e^{[\gamma]}\right)-d_{2}^{-} e^{-\frac{[\gamma]}{2}} \sin \left(\frac{\sqrt{3}}{2}[\gamma]\right)\right] } \\
&+\sum_{\gamma=1}^{\infty} D[N+\gamma+1] \cdot\left[d_{1}^{+}\left(e^{\frac{1+[\gamma]}{2}} \cos \left(\frac{\sqrt{3}}{2}(1+[\gamma])\right)-e^{\frac{1}{2}} \cos \left(\frac{\sqrt{3}}{2}\right) e^{-[\gamma]}\right)\right. \\
&\left.+d_{2}^{+}\left(e^{\frac{1+[\gamma]}{2}} \sin \left(\frac{\sqrt{3}}{2}(1+[\gamma])\right)-e^{\frac{1}{2}} \sin \left(\frac{\sqrt{3}}{2}\right) e^{-[\gamma]}\right)\right] \\
&= {\left[\varphi(0) \sum_{\gamma=1}^{\infty} D[\gamma-1] e^{[\gamma]}+\varphi(1) \sum_{\gamma=1}^{\infty} D[N+\gamma+1] e^{-[\gamma]}+\sum_{\gamma=0}^{N} D[\gamma+1] \varphi[\gamma]\right], } \\
& \sum_{\gamma=1}^{\infty} D[\gamma-2] \cdot\left[d_{1}^{-}\left(e^{-\frac{[\gamma]}{2}} \cos \left(\frac{\sqrt{3}}{2}[\gamma]\right)-e^{[\gamma]}\right)-d_{2}^{-} e^{-\frac{[\gamma]}{2}} \sin \left(\frac{\sqrt{3}}{2}[\gamma]\right)\right] \\
&+ \sum_{\gamma=1}^{\infty} D[N+\gamma+2] \cdot\left[d_{1}^{+}\left(e^{\frac{1+[\gamma]}{2}} \cos \left(\frac{\sqrt{3}}{2}(1+[\gamma])\right)-e^{\frac{1}{2}} \cos \left(\frac{\sqrt{3}}{2}\right) e^{-[\gamma]}\right)\right. \\
&+\left.d_{2}^{+}\left(e^{\frac{1+[\gamma]}{2}} \sin \left(\frac{\sqrt{3}}{2}(1+[\gamma])\right)-e^{\frac{1}{2}} \sin \left(\frac{\sqrt{3}}{2}\right) e^{-[\gamma]}\right)\right] \\
&=- {\left[\varphi(0) \sum_{\gamma=1}^{\infty} D[\gamma-2] e^{[\gamma]}+\varphi(1) \sum_{\gamma=1}^{\infty} D[N+\gamma+2] e^{-[\gamma]}+\sum_{\gamma=0}^{N} D[\gamma+2] \varphi[\gamma]\right] . }
\end{aligned}
$$

Now we consider the cases $\beta=N+1, N+2$. From (34) we obtain

$$
\begin{aligned}
& \sum_{\gamma=1}^{\infty} DN+\gamma+1] \cdot\left[d_{1}^{-}\left(e^{-\frac{[\gamma]}{2}} \cos \left(\frac{\sqrt{3}}{2}[\gamma]\right)-e^{[\gamma]}\right)-d_{2}^{-} e^{-\frac{[\gamma]}{2}} \sin \left(\frac{\sqrt{3}}{2}[\gamma]\right)\right] \\
&+\sum_{\gamma=1}^{\infty} D[\gamma-1] \cdot\left[d_{1}^{+}\left(e^{\frac{1+[\gamma]}{2}} \cos \left(\frac{\sqrt{3}}{2}(1+[\gamma])\right)-e^{\frac{1}{2}} \cos \left(\frac{\sqrt{3}}{2}\right) e^{-[\gamma]}\right)\right. \\
&\left.+d_{2}^{+}\left(e^{\frac{1+[\gamma]}{2}} \sin \left(\frac{\sqrt{3}}{2}(1+[\gamma])\right)-e^{\frac{1}{2}} \sin \left(\frac{\sqrt{3}}{2}\right) e^{-[\gamma]}\right)\right] \\
&=- {\left[\varphi(0) \sum_{\gamma=1}^{\infty} D[N+\gamma+1] e^{[\gamma]}+\varphi(1) \sum_{\gamma=1}^{\infty} D[\gamma-1] e^{-[\gamma]}\right.} \\
&\left.+\sum_{\gamma=0}^{N} D[N+1-\gamma] \varphi[\gamma]\right], \\
& \sum_{\gamma=1}^{\infty} D[N+\gamma+2] \cdot\left[d_{1}^{-}\left(e^{-\frac{[\gamma]}{2}} \cos \left(\frac{\sqrt{3}}{2}[\gamma]\right)-e^{[\gamma]}\right)-d_{2}^{-} e^{-\frac{[\gamma]}{2}} \sin \left(\frac{\sqrt{3}}{2}[\gamma]\right)\right] \\
&+\sum_{\gamma=1}^{\infty} D[\gamma-2] \cdot\left[d_{1}^{+}\left(e^{\frac{1+[\gamma]}{2}} \cos \left(\frac{\sqrt{3}}{2}(1+[\gamma])\right)-e^{\frac{1}{2}} \cos \left(\frac{\sqrt{3}}{2}\right) e^{-[\gamma]}\right)\right. \\
&+\left.d_{2}^{+}\left(e^{\frac{1+[\gamma]}{2}} \sin \left(\frac{\sqrt{3}}{2}(1+[\gamma])\right)-e^{\frac{1}{2}} \sin \left(\frac{\sqrt{3}}{2}\right) e^{-[\gamma]}\right)\right]
\end{aligned}
$$




$$
\begin{aligned}
= & -\left[\varphi(0) \sum_{\gamma=1}^{\infty} D[N+\gamma+2] e^{[\gamma]}+\varphi(1) \sum_{\gamma=1}^{\infty} D[\gamma-2] e^{-[\gamma]}\right. \\
& \left.+\sum_{\gamma=0}^{N} D[N+2-\gamma] \varphi[\gamma]\right]
\end{aligned}
$$

Thus for unknowns $d_{1}^{-}, d_{1}^{+}, d_{2}^{-}$, and $d_{2}^{+}$we get system (35)-(38) consisting of four linear equations. Since the interpolation problem has a unique solution, the main matrix of this system is nonsingular. To find the unknowns $d_{1}^{-}, d_{1}^{+}, d_{2}^{-}$, and $d_{2}^{+}$, we rewrite system (35)(38) as follows:

$$
\left\{\begin{array}{l}
M_{11} d_{1}^{-}+M_{12} d_{2}^{-}+M_{13} d_{1}^{+}+M_{14} d_{2}^{+}=T_{1}, \\
M_{21} d_{1}^{-}+M_{22} d_{2}^{-}+M_{23} d_{1}^{+}+M_{24} d_{2}^{+}=T_{2}, \\
M_{31} d_{1}^{-}+M_{32} d_{2}^{-}+M_{33} d_{1}^{+}+M_{34} d_{2}^{+}=T_{3}, \\
M_{41} d_{1}^{-}+M_{42} d_{2}^{-}+M_{43} d_{1}^{+}+M_{44} d_{2}^{+}=T_{4}
\end{array}\right.
$$

where

$$
\begin{aligned}
& M_{11}=\frac{3}{K}\left[\left(K_{3}-K_{1}\right)\left(e^{-\frac{h}{2}} \cos \left(\frac{\sqrt{3}}{2} h\right)-e^{h}\right)+e^{-h} \cos (\sqrt{3} h)-e^{2 h}\right. \\
& \left.-\sum_{k=1}^{2} \frac{B_{k}}{\lambda_{k}}\left[\frac{\lambda_{k}-e^{\frac{h}{2}} \cos \left(\frac{\sqrt{3}}{2} h\right)}{\lambda_{k}^{2}-2 \lambda_{k} e^{\frac{h}{2}} \cos \left(\frac{\sqrt{3}}{2} h\right)+e^{h}}+\frac{e^{h}}{1-\lambda_{k} e^{h}}\right]\right] \text {, } \\
& M_{12}=-\frac{3}{K}\left[\left(K_{3}-K_{1}\right) \cdot e^{-\frac{h}{2}} \sin \left(\frac{\sqrt{3}}{2} h\right)+e^{-h} \sin (\sqrt{3} h)\right. \\
& \left.+\sum_{k=1}^{2} \frac{B_{k}}{\lambda_{k}} \frac{e^{\frac{h}{2}} \sin \left(\frac{\sqrt{3}}{2} h\right)}{\lambda_{k}^{2}-2 \lambda_{k} e^{\frac{h}{2}} \cos \left(\frac{\sqrt{3}}{2} h\right)+e^{h}}\right] \text {, } \\
& M_{13}=-\frac{3 e^{\frac{1}{2}}}{K} \sum_{k=1}^{2} B_{k} \lambda_{k}^{N+1}\left[\frac{e^{h} \cos \left(\frac{\sqrt{3}}{2}\right) \lambda_{k}-e^{\frac{h}{2}} \cos \left(\frac{\sqrt{3}}{2}(1+h)\right)}{e^{h} \lambda_{k}^{2}-2 \lambda_{k} e^{\frac{h}{2}} \cos \left(\frac{\sqrt{3}}{2} h\right)+1}-\frac{\cos \left(\frac{\sqrt{3}}{2}\right)}{\lambda_{k}-e^{h}}\right] \text {, } \\
& M_{14}=-\frac{3 e^{\frac{1}{2}}}{K} \sum_{k=1}^{2} B_{k} \lambda_{k}^{N+1}\left[\frac{e^{h} \sin \left(\frac{\sqrt{3}}{2}\right) \lambda_{k}-e^{\frac{h}{2}} \sin \left(\frac{\sqrt{3}}{2}(1+h)\right)}{e^{h} \lambda_{k}^{2}-2 \lambda_{k} e^{\frac{h}{2}} \cos \left(\frac{\sqrt{3}}{2} h\right)+1}-\frac{\sin \left(\frac{\sqrt{3}}{2}\right)}{\lambda_{k}-e^{h}}\right] \text {, } \\
& M_{21}=-\frac{3}{K} \sum_{k=1}^{2} B_{k} \lambda_{k}^{N+1}\left[\frac{\lambda_{k}-e^{\frac{h}{2}} \cos \left(\frac{\sqrt{3}}{2} h\right)}{\lambda_{k}^{2}-2 \lambda_{k} e^{\frac{h}{2}} \cos \left(\frac{\sqrt{3}}{2} h\right)+e^{h}}+\frac{e^{h}}{1-e^{h} \lambda_{k}}\right] \text {, } \\
& M_{22}=-\frac{3}{K} \sum_{k=1}^{2} B_{k} \lambda_{k}^{N+1} \frac{e^{\frac{h}{2}} \sin \left(\frac{\sqrt{3}}{2} h\right)}{\lambda_{k}^{2}-2 \lambda_{k} e^{\frac{h}{2}} \cos \left(\frac{\sqrt{3}}{2} h\right)+e^{h}}, \\
& M_{23}=\frac{3 e^{\frac{1}{2}}}{K}\left[\left(K_{3}-K_{1}\right)\left(e^{\frac{h}{2}} \cos \left(\frac{\sqrt{3}}{2}(1+h)\right)-\cos \left(\frac{\sqrt{3}}{2}\right) e^{-h}\right)+e^{h} \cos \left(\frac{\sqrt{3}}{2}(1+2 h)\right)\right. \\
& \left.-\cos \left(\frac{\sqrt{3}}{2}\right) e^{-2 h}-\sum_{k=1}^{2} \frac{B_{k}}{\lambda_{k}}\left[\frac{e^{h} \cos \left(\frac{\sqrt{3}}{2}\right) \lambda_{k}-e^{\frac{h}{2}} \cos \left(\frac{\sqrt{3}}{2}(1+h)\right)}{e^{h} \lambda_{k}^{2}-2 \lambda_{k} e^{\frac{h}{2}} \cos \left(\frac{\sqrt{3}}{2} h\right)+1}-\frac{\cos \left(\frac{\sqrt{3}}{2}\right)}{\lambda_{k}-e^{h}}\right]\right],
\end{aligned}
$$




$$
\begin{aligned}
& M_{24}=\frac{3 e^{\frac{1}{2}}}{K}\left[\left(K_{3}-K_{1}\right)\left(e^{\frac{h}{2}} \sin \left(\frac{\sqrt{3}}{2}(1+h)\right)-\sin \left(\frac{\sqrt{3}}{2}\right) e^{-h}\right)+e^{h} \sin \left(\frac{\sqrt{3}}{2}(1+2 h)\right)\right. \\
& \left.-\sin \left(\frac{\sqrt{3}}{2}\right) e^{-2 h}-\sum_{k=1}^{2} \frac{B_{k}}{\lambda_{k}}\left[\frac{e^{h} \sin \left(\frac{\sqrt{3}}{2}\right) \lambda_{k}-e^{\frac{h}{2}} \sin \left(\frac{\sqrt{3}}{2}(1+h)\right)}{e^{h} \lambda_{k}^{2}-2 \lambda_{k} e^{\frac{h}{2}} \cos \left(\frac{\sqrt{3}}{2} h\right)+1}-\frac{\sin \left(\frac{\sqrt{3}}{2}\right)}{\lambda_{k}-e^{h}}\right]\right], \\
& M_{31}=\frac{3}{K}\left[\left(K_{3}-K_{1}\right)\left(e^{-h} \cos (\sqrt{3} h)-e^{2 h}\right)+e^{-\frac{h}{2}} \cos \left(\frac{\sqrt{3}}{2} h\right)-e^{h}+e^{-\frac{3}{2} h} \cos \left(\frac{3 \sqrt{3}}{2} h\right)\right. \\
& -e^{3 h}+\left(e^{-\frac{h}{2}} \cos \left(\frac{\sqrt{3}}{2} h\right)-e^{h}\right) \sum_{k=1}^{2} \frac{B_{k}}{\lambda_{k}^{2}}\left(\lambda_{k}^{2}-1\right) \\
& \left.-\sum_{k=1}^{2} \frac{B_{k}}{\lambda_{k}^{2}}\left[\frac{\lambda_{k}-e^{\frac{h}{2}} \cos \left(\frac{\sqrt{3}}{2} h\right)}{\lambda_{k}^{2}-2 \lambda_{k} e^{\frac{h}{2}} \cos \left(\frac{\sqrt{3}}{2} h\right)+e^{h}}+\frac{e^{h}}{1-e^{h} \lambda_{k}}\right]\right] \\
& M_{32}=-\frac{3}{K}\left[\left(K_{3}-K_{1}\right) e^{-h} \sin (\sqrt{3} h)+e^{-\frac{h}{2}} \sin \left(\frac{\sqrt{3}}{2} h\right)+e^{-\frac{3}{2} h} \sin \left(\frac{3 \sqrt{3}}{2} h\right)\right. \\
& \left.+e^{-\frac{h}{2}} \sin \left(\frac{\sqrt{3}}{2} h\right) \sum_{k=1}^{2} \frac{B_{k}}{\lambda_{k}^{2}}\left(\lambda_{k}^{2}-1\right)+\sum_{k=1}^{2} \frac{B_{k}}{\lambda_{k}^{2}} \frac{e^{\frac{h}{2}} \sin \left(\frac{\sqrt{3}}{2} h\right)}{\lambda_{k}^{2}-2 \lambda_{k} e^{\frac{h}{2}} \cos \left(\frac{\sqrt{3}}{2} h\right)+e^{h}}\right], \\
& M_{33}=-\frac{3 e^{\frac{1}{2}}}{K} \sum_{k=1}^{2} B_{k} \lambda_{k}^{N+2}\left[\frac{e^{h} \cos \left(\frac{\sqrt{3}}{2}\right) \lambda_{k}-e^{\frac{h}{2}} \cos \left(\frac{\sqrt{3}}{2}(1+h)\right)}{e^{h} \lambda_{k}^{2}-2 \lambda_{k} e^{\frac{h}{2}} \cos \left(\frac{\sqrt{3}}{2} h\right)+1}-\frac{\cos \left(\frac{\sqrt{3}}{2}\right)}{\lambda_{k}-e^{h}}\right] \text {, } \\
& M_{34}=-\frac{3 e^{\frac{1}{2}}}{K} \sum_{k=1}^{2} B_{k} \lambda_{k}^{N+2}\left[\frac{e^{h} \sin \left(\frac{\sqrt{3}}{2}\right) \lambda_{k}-e^{\frac{h}{2}} \sin \left(\frac{\sqrt{3}}{2}(1+h)\right)}{e^{h} \lambda_{k}^{2}-2 \lambda_{k} e^{\frac{h}{2}} \cos \left(\frac{\sqrt{3}}{2} h\right)+1}-\frac{\sin \left(\frac{\sqrt{3}}{2}\right)}{\lambda_{k}-e^{h}}\right] \text {, } \\
& M_{41}=-\frac{3}{K} \sum_{k=1}^{2} B_{k} \lambda_{k}^{N+2}\left[\frac{\lambda_{k}-e^{\frac{h}{2}} \cos \left(\frac{\sqrt{3}}{2} h\right)}{\lambda_{k}^{2}-2 \lambda_{k} e^{\frac{h}{2}} \cos \left(\frac{\sqrt{3}}{2} h\right)+e^{h}}+\frac{e^{h}}{1-e^{h} \lambda_{k}}\right] \text {, } \\
& M_{42}=-\frac{3}{K} \sum_{k=1}^{2} B_{k} \lambda_{k}^{N+2} \frac{e^{\frac{h}{2}} \sin \left(\frac{\sqrt{3}}{2} h\right)}{\lambda_{k}^{2}-2 \lambda_{k} e^{\frac{h}{2}} \cos \left(\frac{\sqrt{3}}{2} h\right)+e^{h}}, \\
& M_{43}=\frac{3 e^{\frac{1}{2}}}{K}\left[\left(K_{3}-K_{1}\right)\left(e^{h} \cos \left(\frac{\sqrt{3}}{2}(1+2 h)\right)-\cos \left(\frac{\sqrt{3}}{2}\right) e^{-2 h}\right)+e^{\frac{h}{2}} \cos \left(\frac{\sqrt{3}}{2}(1+h)\right)\right. \\
& -\cos \left(\frac{\sqrt{3}}{2}\right) e^{-h}+e^{\frac{3}{2} h} \cos \left(\frac{\sqrt{3}}{2}(1+3 h)\right)-\cos \left(\frac{\sqrt{3}}{2}\right) e^{-3 h} \\
& +\left(e^{\frac{h}{2}} \cos \left(\frac{\sqrt{3}}{2}(1+h)\right)-\cos \left(\frac{\sqrt{3}}{2}\right) e^{-h}\right) \sum_{k=1}^{2} \frac{B_{k}}{\lambda_{k}^{2}}\left(\lambda_{k}^{2}-1\right) \\
& \left.-\sum_{k=1}^{2} \frac{B_{k}}{\lambda_{k}^{2}}\left[\frac{e^{h} \cos \left(\frac{\sqrt{3}}{2}\right) \lambda_{k}-e^{\frac{h}{2}} \cos \left(\frac{\sqrt{3}}{2}(1+h)\right)}{e^{h} \lambda_{k}^{2}-2 \lambda_{k} e^{\frac{h}{2}} \cos \left(\frac{\sqrt{3}}{2} h\right)+1}-\frac{\cos \left(\frac{\sqrt{3}}{2}\right)}{\lambda_{k}-e^{h}}\right]\right] \text {, } \\
& M_{44}=\frac{3 e^{\frac{1}{2}}}{K}\left[\left(K_{3}-K_{1}\right)\left(e^{h} \sin \left(\frac{\sqrt{3}}{2}(1+2 h)\right)-\sin \left(\frac{\sqrt{3}}{2}\right) e^{-2 h}\right)+e^{\frac{h}{2}} \sin \left(\frac{\sqrt{3}}{2}(1+h)\right)\right. \\
& -\sin \left(\frac{\sqrt{3}}{2}\right) e^{-h}+e^{\frac{3}{2} h} \sin \left(\frac{\sqrt{3}}{2}(1+3 h)\right)-\sin \left(\frac{\sqrt{3}}{2}\right) e^{-3 h} \\
& +\left(e^{\frac{h}{2}} \sin \left(\frac{\sqrt{3}}{2}(1+h)\right)-\sin \left(\frac{\sqrt{3}}{2}\right) e^{-h}\right) \sum_{k=1}^{2} \frac{B_{k}}{\lambda_{k}^{2}}\left(\lambda_{k}^{2}-1\right)
\end{aligned}
$$




$$
\begin{aligned}
&\left.-\sum_{k=1}^{2} \frac{B_{k}}{\lambda_{k}^{2}}\left[\frac{e^{h} \sin \left(\frac{\sqrt{3}}{2}\right) \lambda_{k}-e^{\frac{h}{2}} \sin \left(\frac{\sqrt{3}}{2}(1+h)\right)}{e^{h} \lambda_{k}^{2}-2 \lambda_{k} e^{\frac{h}{2}} \cos \left(\frac{\sqrt{3}}{2} h\right)+1}-\frac{\sin \left(\frac{\sqrt{3}}{2}\right)}{\lambda_{k}-e^{h}}\right]\right], \\
& T_{1}= \frac{3}{K}\left[\sum_{k=1}^{2} B_{k} \sum_{\gamma=0}^{N} \lambda_{k}^{\gamma} \varphi(h \gamma)+\varphi(0)\left[1+\left(K_{3}-K_{1}\right) e^{h}+e^{2 h}+\sum_{k=1}^{2} \frac{B_{k} e^{h}}{\lambda_{k}-e^{h} \lambda_{k}^{2}}\right]\right. \\
&\left.+\varphi(1) \sum_{k=1}^{2} \frac{B_{k} \lambda_{k}^{N+1}}{e^{h}-\lambda_{k}}\right], \\
& T_{2}= \frac{3}{K}\left[\sum_{k=1}^{2} B_{k} \sum_{\gamma=0}^{N} \lambda_{k}^{N-\gamma} \varphi(h \gamma)+\varphi(0) \sum_{k=1}^{2} \frac{e^{h} B_{k} \lambda_{k}^{N+1}}{1-e^{h} \lambda_{k}}+\varphi(1)\left[1+\left(K_{3}-K_{1}\right) e^{-h}\right.\right. \\
&\left.\left.+e^{-2 h}-\sum_{k=1}^{2} \frac{B_{k}}{\lambda_{k}^{2}-e^{h} \lambda_{k}}\right]\right], \\
& T_{3}= \frac{3}{K}\left[\sum_{k=1}^{2} B_{k} \sum_{\gamma=0}^{N} \lambda_{k}^{\gamma+1} \varphi(h \gamma)+\varphi(0)\left[\left(K_{3}-K_{1}\right) e^{2 h}+e^{h}+e^{3 h}\right.\right. \\
&\left.\left.+e^{h} \sum_{k=1}^{2} \frac{B_{k}}{\lambda_{k}^{2}}\left(\lambda_{k}^{2}-1\right)+\sum_{k=1}^{2} \frac{B_{k} e^{h}}{\lambda_{k}^{2}-e^{h} \lambda_{k}^{3}}\right]+\varphi(1) \sum_{k=1}^{2} \frac{B_{k} \lambda_{k}^{N+2}}{e^{h}-\lambda_{k}}\right], \\
& T_{4}= \frac{3}{K}\left[\sum_{k=1}^{2} B_{k} \sum_{\gamma=0}^{N} \lambda_{k}^{N+1-\gamma} \varphi(h \gamma)+\varphi(0) \sum_{k=1}^{2} \frac{e^{h} B_{k} \lambda_{k}^{N+2}}{1-e^{h} \lambda_{k}}+\varphi(1)\left[\left(K_{3}-K_{1}\right) e^{-2 h}\right.\right. \\
&\left.\left.+e^{-h}+e^{-3 h}+e^{-h} \sum_{k=1}^{2} \frac{B_{k}}{\lambda_{k}^{2}}\left(\lambda_{k}^{2}-1\right)-\sum_{k=1}^{2} \frac{B_{k}}{\lambda_{k}^{3}-e^{h} \lambda_{k}^{2}}\right]\right], \\
&
\end{aligned}
$$

where $\left|\lambda_{k}\right|<1$.

Solving system (39), we find $d_{1}^{-}, d_{2}^{-}, d_{1}^{+}, d_{2}^{+}$. Then using (32) and (33), we find $d_{0}^{-}$and $d_{0}^{+}$. From (21), for $\beta=0,1, \ldots, N$, we deduce

$$
\begin{aligned}
C[\beta]= & \sum_{\gamma=0}^{N} D[\beta-\gamma] \varphi[\gamma]+\sum_{\gamma=1}^{\infty} D[\beta+\gamma]\left[d_{0}^{-} e^{[\gamma]}+d_{1}^{-} e^{-\frac{[\gamma]}{2}} \cos \left(\frac{\sqrt{3}}{2}[\gamma]\right)\right. \\
& \left.-d_{2}^{-} e^{-\frac{[\gamma]}{2}} \sin \left(\frac{\sqrt{3}}{2}[\gamma]\right)\right]+\sum_{\gamma=1}^{\infty} D[N+\gamma-\beta]\left[d_{0}^{+} e^{-(1+[\gamma])}\right. \\
& \left.+d_{1}^{+} e^{\frac{1+[\gamma]}{2}} \cos \left(\frac{\sqrt{3}}{2}(1+[\gamma])\right)+d_{2}^{+} e^{\frac{1+[\gamma]}{2}} \sin \left(\frac{\sqrt{3}}{2}(1+[\gamma])\right)\right]
\end{aligned}
$$

from which, using (14) and taking into account (30) and (31), after some calculations, we arrive at the expressions for the coefficients $C[\beta], \beta=0,1, \ldots, N$, which are given in the statement of the theorem.

Theorem 3 is proved.

\section{Numerical results}

In this section, we give some numerical results using Theorem 3.

In numerical examples,we consider the functions $f_{1}(x)=e^{x}$ and $f_{2}(x)=\sin x$. We denote the corresponding interpolation splines of the form $(8)$ by $S\left(f_{1} ; x\right)$ and $S\left(f_{2} ; x\right)$, respectively. 

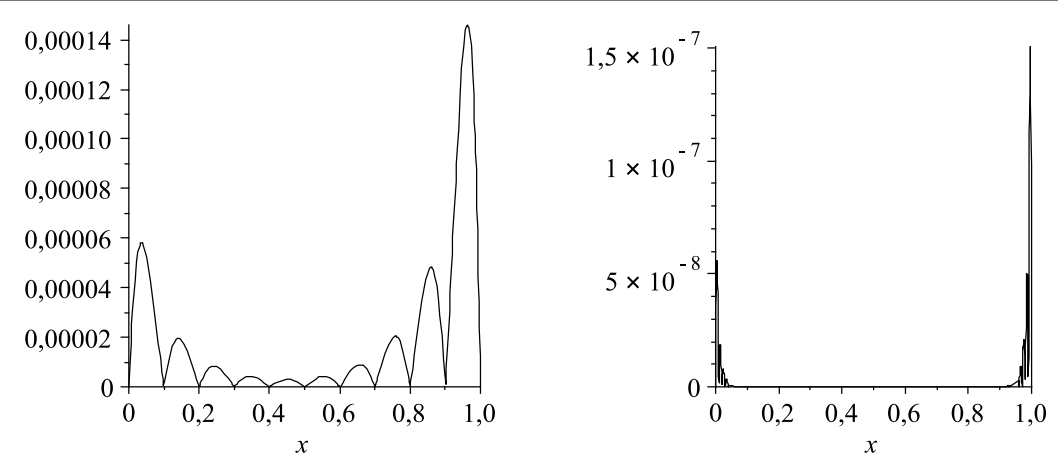

Figure 1 Graphs of absolute errors $\left|f_{1}(x)-S\left(f_{1} ; x\right)\right|$ for $N=10$ (left) and $N=100$ (right)
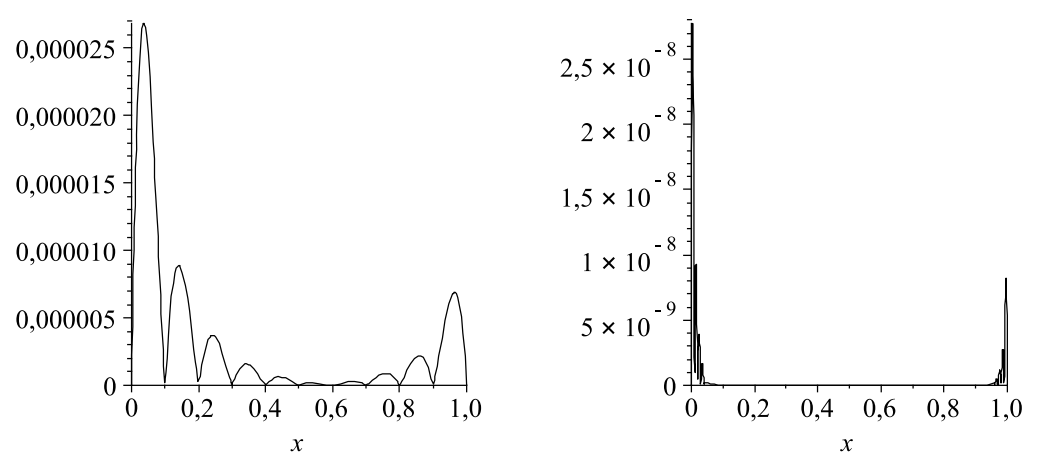

Figure 2 Graphs of absolute errors $\left|f_{2}(x)-S\left(f_{2} ; x\right)\right|$ for $N=10$ (left) and $N=100$ (right)

Applying $S(x)$ with $N=10$ and $N=100$ and using Theorem 3 for the functions $f_{1}(x)$ and $f_{2}(x)$, we obtain the absolute errors. The graphs of the corresponding absolute errors are displayed in Figs. 1 and 2. We can see that by increasing the value of $N$ the absolute errors between interpolation splines and the given functions decrease and the order of convergence of the interpolation formula (8) in these examples is $O\left(h^{3}\right)$.

\section{Acknowledgements}

The authors are very thankful to professor A.R. Hayotov for discussion of the results and for some bibliographic references. The authors are also very grateful to reviewers for remarks and suggestions.

\section{Funding}

Not applicable.

Availability of data and materials

Not applicable.

\section{Competing interests}

The authors declare that they have no competing interests.

Authors' contributions

Both authors contributed equally to the writing of this paper. Both authors read and approved the manuscript.

\section{Publisher's Note}

Springer Nature remains neutral with regard to jurisdictional claims in published maps and institutional affiliations.

Received: 8 April 2020 Accepted: 29 June 2020 Published online: 14 July 2020 


\section{References}

1. Ahlberg, J.H., Nilson, E.N., Walsh, J.L.: The Theory of Splines and Their Applications. Mathematics in Science and Engineering. Academic Press, New York (1967)

2. Boltaev, A.K., Nuraliev, F.A.: On a discrete analogue of a differential operator. Probl. Comput. Appl. Math. 20(2), 71-78 (2019)

3. Boltaev, A.K., Shadimetov, Kh.M.: The discrete analogue of the differential operator $\frac{\mathrm{d}^{6}}{\mathrm{~d} x^{6}}-1$. Uzbek. Math. J. 3, 209-216 (2011)

4. Cabada, A., Hayotov, A.R., Shadimetov, Kh.M.: Construction of $D^{m}$-splines in $L_{2}^{(m)}(0,1)$ space by Sobolev method. Appl. Math. Comput. 244, 542-551 (2014)

5. Hayotov, A.R.: Construction of interpolation splines minimizing the semi-norm in the space $K_{2}\left(P_{m}\right)$. J. Sib. Fed. Univ. Math. Phys. 11(3), 383-396 (2018)

6. Hayotov, A.R., Milovanović, G.V., Shadimetov, Kh.M.: Interpolation splines minimizing a semi-norm. Calcolo 51(2), 245-260 (2014)

7. Hayotov, A.R., Milovanović, G.V., Shadimetov, Kh.M.: Optimal quadrature formulas and interpolation splines minimizing the semi-norm in the Hilbert space $K_{2}\left(P_{2}\right)$. In: Milovanović, G.V., Rassias, M.T. (eds.) Analytic Number Theory, Approximation Theory, and Special Functions, pp. 572-611 (2014)

8. Jena, M.K., Shunmugaraj, P., Das, P.C.: A subdivision algorithm for trigonometric spline curves. Comput. Aided Geom. Des. 19, 71-88 (2002)

9. Jia, R.-Q.: On local linear functionals for L-splines. J. Approx. Theory 33, 96-110 (1981)

10. Koch, P.E.: Multivariate trigonometric B-splines. J. Approx. Theory 54, 162-168 (1988)

11. Koch, P.E., Lyche, T.: Interpolation with exponential B-splines in tension. Computing 8, 173-190 (1993)

12. Koch, P.E., Lyche, T., Neamtu, M., Schumaker, L.L.: Control curves and knot insertion for trigonometric splines. Adv. Comput. Math. 3, 405-424 (1995)

13. Kvasov, B.I., Sattayatham, P: GB-Splines of arbitrary order. J. Comput. Appl. Math. 104, $63-88$ (1999)

14. Lempel, A., Seroussi, G.: Systematic derivation of spline bases. Comput. Aided Geom. Des. 9, 349-362 (1992)

15. Lu, Y., Wang, G., Yang, X.: Uniform hyperbolic polynomial B-spline curves. Comput. Aided Geom. Des. 19, 379-393 (2002)

16. Lyche, T., Schumaker, L.L., Stanley, S.: Quasi-interpolants based on trigonometric splines. J. Approx. Theory 95, 280-309 (1998)

17. McCartin, B.J.: Theory of exponential splines. J. Approx. Theory 66, 1-23 (1991)

18. Rogina, M.: On construction of fourth order Chebyshev splines. Math. Commun. 4, 83-92 (1999)

19. Sakai, M., Togashi, A.: On hyperbolic and trigonometric B-splines on equally spaced knots. Rep. Fac. Sci. Kagoshima Univ. Math. Phys. Chem. 23, 13-21 (1990)

20. Schoenberg, I.J.: On trigonometric spline interpolation. J. Math. Mech. 13, 795-825 (1964)

21. Schumaker, L.L:: On hyperbolic splines. J. Approx. Theory 38, 144-166 (1983)

22. Schumaker, L.L.: Spline Functions: Basic Theory. Cambridge University Press, Cambridge (2007)

23. Shadimetov, Kh.M., Hayotov, A.R.: Construction of interpolation splines minimizing semi-norm in $W_{2}^{(m, m-1)}(0,1)$ space. BIT Numer. Math. 53(2), 545-563 (2013)

24. Shadimetov, Kh.M., Hayotov, A.R., Azamov, S.S.: Interpolation splines minimizing semi-norm in $K_{2}\left(P_{2}\right)$ space. Am. J. Numer. Anal. 2(4), 107-114 (2014). https://doi.org/10.12691/aina-2-4-3

25. Sobolev, S.L.: Introduction to the Theory of Cubature Formulas. Nauka, Moscow (1974) (in Russian)

26. Sobolev, S.L.: The coefficients of optimal quadrature formulas. In: Selected Works of S.L. Sobolev, pp. 561-566. Springer, Berlin (2006)

27. Sobolev, S.L., Vaskevich, V.L.: The Theory of Cubature Formulas. Kluwer Academic, Dordrecht (1997)

28. Vasilenko, V.A.: Spline Functions: Theory, Algorithms, Programs. Nauka, Novosibirsk (1983) (in Russian)

29. Vladimirov, V.S.: Generalized Functions in Mathematical Physics. Mir, Moscow (1983) (in Russian)

30. Walz, G.: Identities for trigonometric B-splines with an application to curve design. BIT Numer. Math. 37, 189-201 (1997)

31. Zhang, J.: C-curves, an extension of cubic curves. Comput. Aided Geom. Des. 13, 199-217 (1996)

32. Zhang, J.: Two different forms of C-B-splines. Comput. Aided Geom. Des. 14, 31-41 (1997)

\section{Submit your manuscript to a SpringerOpen ${ }^{\circ}$ journal and benefit from:}

- Convenient online submission

- Rigorous peer review

- Open access: articles freely available online

- High visibility within the field

- Retaining the copyright to your article

Submit your next manuscript at $\gg$ springeropen.com 